\title{
Robotic Service Cores for Ambient Assisted Living
}

\author{
Matthias Kranz, Thomas Linner, Bernhard Ellmann, Andreas Bittner, Luis Roalter \\ Technische Universität München \\ Arcisstrasse 21, 80333 München \\ Germany \\ matthias.kranz@tum.de, \{thomas.linner, bernhard.ellmann, andreas.bittner\}@bri.ar.tum.de, roalter@tum.de
}

\begin{abstract}
In the paper we present the conceptual idea of scalable robotic service cores for a simplified and rapid deployment of ambient assisted living environments. We situate our approach in between fully networked homes on the one hand and smart artifacts on the other end of the scale. We elaborate on the multidimensional interdisciplinary challenges that need to be addressed for successful future pervasive healthcare systems. We illustrate our approach at the instance of a functional scale model of an example for a robotic service core and situate it in the body of research in the dimensions healthcare, architecture, design, society, culture and pervasive computing.
\end{abstract}

Keywords-Pervasive Healthcare, Ambient Assisted Living, Service Units

\section{INDEPENDENT LIVING AT HOME}

As shown in many analyses and studies, an extended stay of elderly people in an appropriate and with assistive technologies equipped home environment is less costly, and in most cases also more effective than a stationary stay in a care or nursing facility. The continuance of activity in naturally grown environments and social networks helps effectively to prevent somatic, motor, mental and social disorders, when customized and personalized health environments meeting multiple requirements are installed around the care receivers. Moreover, local governments and especially the housing industry are interested in elderly people living in their homes supported by integrated sets of classical methods and pervasive technologies in order to keep up the related value creation and to establish new value systems. By our work, we wish to contribute to this important societal goal.

The challenges of facilitating independent living at home with integrated sets of methods and technologies require a holistic and interdisciplinary approach, including experts from the domains of medical science, architecture, design, robotics, computer science, electrical engineering, human-computer interaction and the housing industry.

The remainder of the paper is structured as follows: in Sec. II we discuss the domain challenges at the example of the robotic service cores. We continue in Sec. III with the presentation of the instance of a modern, state-of-the-art example of an implementation of a pervasive health system. We present the current state of the demonstrator in Sec. IV: The paper is concluded in Sec. V. with an outlook on future research activities targeted at the development and deployment of the system for future field studies. The discussion of related work is included in the individual sections of this work.

\section{Challenges for Pervasive HealthCARE}

Designing assistive pervasive healthcare systems is a multidimensional, interdisciplinary challenge. These challenges, which we illustrate with examples from current research efforts, have to be considered throughout the complete development process. The intersecting research fields are visualized in Figure 4.

\section{A. Geriatric and Medical Challenges}

The geriatric profile of elderly people in general can be specified by so called "multi-morbidity" [1], which is defined as the co-existence of two or more chronic conditions. All "use cases" are a complex mixture of multiple geriatric requirements as support of daily physical activity, support of mobility, compensate deficits concerning hearing and seeing, support of cognitive abilities, support of emotional and psychological state, support of social interaction or emergency support also require a multiple set of technologies. Some of those requirements can be met with higher efficiency by "passive" systems (architecture, design, barrier free concepts, etc.), some by "active" systems (pervasive health technologies: micro systems technology, sensors, actors, integrated robotic systems, information and communication technology). A carefully chosen and aware combination of both passive and active systems would enhance the ability of environments to holistically address geriatric challenges significantly. All in all, multidimensional component systems that can be customized and even personalized to specific multidimensional needs are gradually becoming a basic requirement: upcoming "personalized healthcare" [5] demands to guarantee the individual care receivers' optimized physical and mental health.

\section{B. Architectural Challenges}

When we talk about "assistive" environments which incorporate pervasive computing technology, we do not mean the summation of independent stand-alone health applications which have the ability to be used at home. For example, a blood pressure measure system, an emergency monitoring system and a "health phone" which are added to an environment, but not networked only generate output within their own area. In those cases the potential of the employed technologies is not used to its full extent. In an "intelligent" or "assistive" environment, those components are more networked than independent and ideally moreover connected to home automation systems, fire detectors, lightning and 
other "modules" or "systems" defined by architecture. Yet, how can we - from that point of view - integrate pervasive computing technology into existing homes, where their deployment would be demanded [2]? It can be assumed that people, who become "elderly" during the next decades in most cases, do not own a "networked" flat or house, providing the necessary infrastructure. Thus, new methods have to be found which allow an efficient transformation of existing homes into networked assistive environments for independent living.

\section{Design Challenges}

The sustainable transformation of existing "low-tech", ordinary homes into assistive technology enhanced home environments suitable for elderly people implicates several challenges concerning design and overall product architectures.

First, it is important to introduce hierarchical modularity or platform strategies cutting cross all related disciplines, so that various assistive technologies or modules can be customized to a specific use case and further be exchanged or extended to meet the variety of possible use cases, even changing over time [3]. The individual modules of the system have to be preconfigurable, easy to install and have to integrate in an existing, presumably non-pervasive computing, home. Maintenance, for care givers and receivers, has to be addressed by a designed-in serviceability. Secondly, the distribution and arrangement of functionalities, as for example kitchen, bath, sleeping and related appliances and technologies have to be considered. We show and discuss this combination later in the paper (see Figures 1 and 2) and present a scale model facilitating this combination. Elderly people tend to have a restricted degree of freedom in mobility and the distribution of mostly needed or life supporting functionalities over several rooms as in most homes today is not compulsorily an optimal solution. Thirdly, a sustainable technology enhanced environment has to hide the complexity of its subsystems [4] to a maximum extent to take mental load and stress from the users as care receivers and to motivate them for activity and interaction with the technology. Persuasiveness here is a key aspect: encouraging activity or a healthy way of living leads to more satisfactory experience. Pervasive health technologies have, following the vision of calm computing, to be integrated in the design of the environment seamlessly, working invisibly in the background. Further, for the communication of information to care receivers, accepted devices as televisions, should be used as primary interfaces, as they state perfect intersections between well known common environments and new pervasive technology network overlays. While not many elderly people today will be accustomed to the use of modern mobile phone technology, they are very well experienced of using a TV set, thus presenting information as overlay ("Please take your pills") seems an obvious approach. Additionally, cultural backgrounds have to be acknowledged. While it is more common, e.g. to accept assistive robots in Asia, this may be different in Europe or the US. Similar, the extent of potential assistive functionality has to be carefully evaluated for the individual target groups of care receivers.

\section{Pervasive Computing Challenges}

Assistive environments need to be flexible and versatile to cope with the dynamically changing requirements of their inhabitants and support them, especially when health- and agerelated disabilities arise. Components of a service unit might be exchanged to reflect the change in the health status, and therefore a pervasive computing middleware system is required to be able to deal with this change. Our approach is presented at the example of a scale model in Sec. IV.

While smart homes such as the AwareHome [8] or PlaceLab [7] have been subject to research, they exemplarily stand for the goal to instrument and equip the whole building or apartment. Our approach allows incremental addition and replacement of modules and components, not requiring an effort comparable to the augmentation of a complete home. This allows costs, as major factor when investigating deployment scenarios with real homes, to be kept within much lower boundaries and to extend the system, when and if necessary.

While MIT's House_n [9] also includes modular intelligent furniture, their approach is on a smaller scale: the cabinets do not necessarily have to form a dedicated ecology e.g. to holistically support a specific need, as proposed by our core service modules. Their systems are not envisioned to form a self-contained room, e.g. supporting only the specific needs of an inhabitant due to his personal state of health. While modular cabinets are of value, esp. w.r.t. general pervasive computing environments, it has to be considered as incomplete when it comes to support assistive or independent living. We therefore argue that dedicated, targeted approach supporting incremental extension of assistive environments is necessary. This has also to be reflected in the choice of middleware.

While we, in this paper, focus on environmental based ambient assisted living and pervasive healthcare, the inclusion of external devices has to be accounted for. External here has two dimensions: external in the sense of physical space, that is outside of the service units such as wearable health monitoring devices and external in the sense that they can come from other manufacturers [10], such as an activity fostering chair for elderly people or a kitchen tool supporting cooking activities [14]. A suitable middleware ideally supports (open) standards and is supported by a large community [11].

\section{E. Social Challenges}

As the computer-human interface (CHI), putting the human at the center, is, besides functionality, the most crucial part regarding the acceptance of any (digital) system, the presentation of and interaction with information has to be integrated already in the development process at an early stage [12]. This also includes the possibility to simulate and predict movement and interaction times, e.g. to move from one spot of interactivity to another on a macroscopic scale, but also the time necessary to perform a gesture in space as input to a system. At least as important as simulating communication networks is therefore the simulation of physicality.

While technology, hardware and software, are necessary to acquire physical phenomena and to make sense of sensor data, it is as crucial to not only connect technology, but humans family members, care givers and care receivers. 
This includes devices such as the CareNet Display [13], but also social networks such as Twitter or the Internet of Things. Ideally, a pervasive middleware not only connects devices to the Internet of Things, but also humans - care givers, care receivers and families - to a "web of support".

Different middleware systems, such as GAIA [17] and MundoCore [15], have been proposed and used in the relatively young research field of pervasive and ubiquitous computing. The challenges of distributed multimodal information processing connecting heterogeneous input and output technologies have very different demands towards middleware systems. Unfortunately, reuse and finally development in this domain is limited usually to the initial developers of a respective middleware and no community yet evolved to pursue the ambitious goal of a unified middleware. Existing systems, therefore, have not been designed to have a long life cycle and to allow for future inclusion of demands and upcoming technologies.

We, therefore, consider the middleware as an extremely important issue towards deployable and working systems. We will later in this paper describe how we hope to have successfully tackled this issue.

\section{F. Cultural challenges}

In Japan, for a long time now, pervasive technologies and especially service robotics have been accounted as basic and natural elements in addressing the problem of the ageing society. Therefore, today several robots are under advanced development which can communicate ("PaPeRo", NEC; "Mamuro", Center of IRT), interact with human beings ("Emiew", Hitachi; "Wakamura", Mitsubishi) or perform complex service tasks ("Hospi", Matsushita). Moreover, obvious robotic subsystems as robotic hands ("Tendy-one", Waseda University), robotic suits ("HAL", Cyberdyne) and robot control interfaces ("Brain Machine Interface", Honda) are basic issues of both scientific and commercial research. A common characteristic of the Japanese efforts is that most service solutions are intentionally designed as "humanoid" or "mobile" systems as obvious robotic systems are widely accepted and demanded by care receivers as well as care givers.

Yet, in Europe, a different strategy to successfully introduce high-tech based services, health or rehabilitation technologies is necessary as "visible" robotic systems are strongly related to production and "dull, dirty and dangerous" tasks. Therefore, the robotic service core has been distinctively designed as a seamless "Immobile Robot", distributed and integrated in the service environment as an invisible and highly customizable companion, supporting health- and age-related disabilities with a high degree of autonomy.

\section{SERVICE CORES}

To guarantee a sustainable implementation of pervasive health technologies, a concept of scalable, highly modular and customizable service cores has been derived from the discussed challenges. Moreover, the approach has been designed as a "compact" alternative to fully networked homes and houses.
Recently designed German prototypes of assistive homes as "Haus der Gegenwart" (eng: house of presence) [24] and "Haus der Zukunft" (eng: house of the future) [25] are exemplarily equipped with a variety of networked pervasive technologies, integrated in modern and "expensive" design. Both these homes act as demonstrator and as technology probe, supporting and facilitating discussion, as does our scale model. Nevertheless both approaches do not address topics as modularity or interoperability on physical or digital level. Those approaches would thus require a house to be built from scratch or at least costly renovation. In contrast to the robotic service core system, approaches presented by those prototypes do not allow gradual implementation, simplified "upgrading" or continuous customization of existing environments, which could be accounted as essential to deploy pervasive health environments as discussed before. Other examples for assistive homes as the "Housen" [26] and the "Toyota PAPI House" [27] offer higher modularity, achieved through modular and open architectural concepts. Yet, they do not fully use the potential of that approach and still require basic parts of the building and its infrastructure to be built from scratch. The scalability of both systems is restricted to newly built environments; they are also not explicitly designed for implementation into existing and naturally grown environments. Although the "Toyota PAPI House" is based on Toyota Home's modular and industrially mass customized unit boxes prefabricated by the so called "skeleton and infill" [27] approach, it is not foreseen that those preconfigured high-tech units could be added to an existing home. The robotic service core addresses these issues integrating pervasive health subsystems through a hierarchically structured and highly scalable prefabricated component system able to be installed as an adaptive continuously changeable subsystem in existing low-tech homes.

\section{A. System Components}

The robotic service core consists of two basic system blocks. First, different service core frames serve as platform or "chassis" in a physical and digital sense (see Figure 7). Secondly, sets of compatible subsystems integrated with pervasive technologies allow customizing the service core to different needs and multidimensional health- and age-related disabilities as discussed before (2.A. Geriatric and Medical Challenges). Variations of the fundamental transition from a normal home to a pervasive healthcare environment stating another mission critical challenge (2.B. Architectural Challenges) are depicted in Figures 1 and 2: the functionality required for a specific care receiver is transferred from different parts of a home to a dedicated, compact and assistive robotic service core.

\section{1) Service Core Types as Integrating Platform}

As of now, independent living in a conventionally designed and low-tech home is often unpleasant and in many cases impossible. It should be noted that, especially when entering a later stage of life, changes of habits and lifestyle - in comparison to previous stages of life - are most dramatically. Also, unforeseen disorders and disorder progresses potentially could make existing houses or flats inapplicable for elderly 
inhabitants from one day to the next. In many cases, a reconfiguration of the existing home or the implementation of various assistive technologies would be needed. Today, this normally is a complex, costly and time consuming matter often forcing elderly people to move. This is where our proposed approach aims to leverage the current situation. The presented approach tries to simplify this process by providing a compact, modular and adaptive service core equipped with exactly the needed assistive technologies. The service core is a highly compact entity which could be implemented in existing flats or homes as an independent sub-module instantly and with minimized efforts. The service core moreover is an integration framework for technologies and it is preconfigured by a service company. The installation time for such a core could be estimated to be less than 2 days. As the service core is expected to be integrated into a care receivers home, his/her well-known and comfortable environment is largely maintained, while at the same time providing the currently required assistive pervasive healthcare technology needed to allow the care receiver to stay in his well-known environment. The modular approach allows substituting and adding additional functionality later if and when needed.

\section{Generally two types of cores could be distinguished:}

Using the Central Type Core various assistive scenarios as well as bath and kitchen functions (which are of high importance concerning many disorders or disabilities) could be arranged compactly around the service core (see Figure 1). The Roomin-room Type Core outlines a even more extreme and compact variant and implements a new "room" as an independent subentity into existing environments (see Figure 2). Both types provide - in contrast to conventional, existing homes - the advantage that all functions needed for independent living can be organized on a minimal space, thus reducing the activity efforts of the elderly to a minimum, meanwhile activity and health condition could be monitored with high precision.

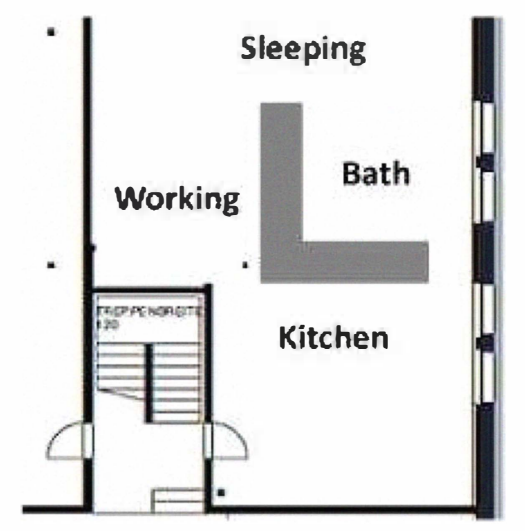

Figure 1. Implementation of Central Core Type: External orientation of functions and related pervasive technologies

\section{2) Service Functions as Subsystems}

For addressing the dicussed design challenges (2.C. Design Challenges), arising when conventional "low-tech" functions should be supplemented with pervasive technologies to an integrated assistive environment, the common practice of environmental design has to be rethought. Any kind of architecture or architectonic space can be defined by hierarchies [27]. In conventional architecture, this means for example that the function of a room is defined by all the rooms' subsystems (walls, windows, furniture, doors), their positioning and the synergies they create. A level above that, the house can be defined as an organization of rooms and floors and so on working together as subsystems to build up the system house as a whole.

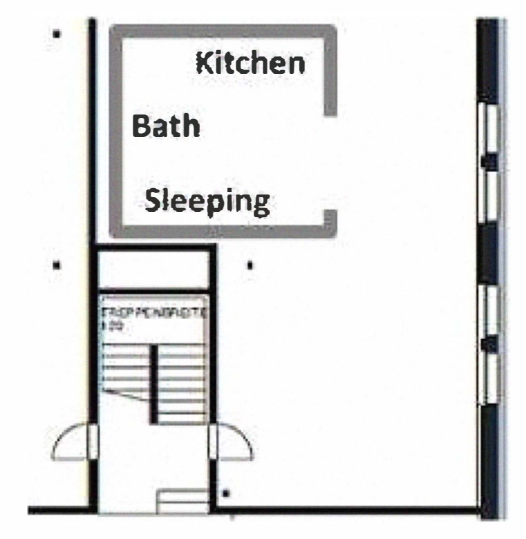

Figure 2. Implementation of Room-in-room Core Type: Internal orientation of functions and related pervasive technologies

From the point of view of a neighborhood area, the houses themselves, streets and other components would then state subsystems which in combination build up the whole system. Thus, in architecture, a systemic method to define functions and spaces exists. Only recently a multitude of new modules equipped or embedded with technologies from the field of micro systems technology and ICT emerge and become part of our living environments. Rooms or walls can be equipped with interface technologies, control components, sensors, actors, assistive technologies and healthcare and wellness systems and a huge number of those systems are under development at the moment - and even more will be developed in the future. To control this new complexity, the above described method of defining spaces or modules or components by their subsystems should not only cover physical entities (walls, windows, furniture, doors etc.), but also a new variety of embedded ICT enabled functionalities and digital services. Up to now, these not-physical functionalities are not an explicit matter in scientific nor in practical architecture. The common practice is still that architecture defines the physical space, which is then later and often by somebody else outfitted with "loose" and not-integrated assistive technologies and/or home automation components. The final functionality, which actually would be built up by an integrative combination of traditional passive systems and new active systems, is not foreseen and explicitly controlled by the architect, as no accepted framework or knowledge about this exists yet.

In order to use the full power and potential of rooms and other physical spaces for supporting "multimorbidity" and multiple requirements, the robotic service core has been based on a new 
language for defining complex pervasive health environments by integrated subsystems.

\section{B. System Performance}

Moreover, the service core, as a system including both platform and compatible service functions has been designed as an invisible and "immobile" robotic system. The use case specific subsystems are not only networked and controllable, but technology works seamlessly and to a high degree autonomously in the environment's background. The robotic service core thus makes use of the concept of distributing and embedding robotic systems into environments.

Environments enriched with distributed sensor-actor-systems, certain autonomy, and able to control their complex internal and external functions are considered as "ImmoBots": Immobile Robots [18]. In general, those systems can cover networked building energy systems as well as power grids or reconfigurable traffic systems. Higher level systems able to coordinate a multitude of internal subsystems whole cities equipped with smart subsystems can be called "Immobile Robots".

\begin{tabular}{|c|c|c|c|}
\hline & Category & Subsystems & $\begin{array}{l}\text { Planning components/ } \\
\text { Scope }\end{array}$ \\
\hline \multirow{2}{*}{\multicolumn{2}{|c|}{ Physical }} & $\begin{array}{l}\text { Building } \\
\text { structure }\end{array}$ & $\begin{array}{l}\text { Bearing structure: steel } \\
\text { concrete, brickwork etc. }\end{array}$ \\
\hline & & Building & Water pipes, cables, air \\
\hline \multirow{3}{*}{\multicolumn{2}{|c|}{$\begin{array}{l}\text { Classical } \\
\text { "passive" } \\
\text { Subsystems }\end{array}$}} & infrastructure & $\begin{array}{l}\text { circulation, energy generating } \\
\text { modules etc. }\end{array}$ \\
\hline & & $\begin{array}{l}\text { Building } \\
\text { modules }\end{array}$ & $\begin{array}{l}\text { Walls, columns, windows, } \\
\text { doors, ceiling etc. }\end{array}$ \\
\hline & & Surfaces & $\begin{array}{l}\text { Painting, stucco, plastering, } \\
\text { textures etc. }\end{array}$ \\
\hline \multirow{9}{*}{\multicolumn{2}{|c|}{$\begin{array}{l}\text { Emerging } \\
\text { "active" } \\
\text { Subsystems }\end{array}$}} & $\begin{array}{l}\text { Mechatronic } \\
\text { systems }\end{array}$ & $\begin{array}{l}\text { Wallcabinet lift, worktopunit } \\
\text { lift, kitchen appliance lift, } \\
\text { liftable toilet }\end{array}$ \\
\hline & & $\begin{array}{l}\text { Embedded } \\
\text { microsystems }\end{array}$ & $\begin{array}{l}\text { Sensors, actors: senor floor, } \\
\text { heat sensors etc., sensors for } \\
\text { health conditions }\end{array}$ \\
\hline & & $\begin{array}{l}\text { Wearable/ } \\
\text { Implanted } \\
\text { Devices }\end{array}$ & $\begin{array}{l}\text { Sensors, actors in the body } \\
\text { area, sensor shirts, implanted } \\
\text { sensors/ actors }\end{array}$ \\
\hline & & $\begin{array}{l}\text { Intelligent } \\
\text { appliances }\end{array}$ & $\begin{array}{l}\text { Controllable lights, } \\
\text { refrigerator, washingmachine }\end{array}$ \\
\hline & & Interfaces & $\begin{array}{l}\text { Touchscreens, voice mail, } \\
\text { communication devices, } \\
\text { mobilphones }\end{array}$ \\
\hline & & Robotics & e.g. robotic bed Panasonic \\
\hline & & Mobility Systems & $\begin{array}{l}\text { Intelligent wheelchairs, } \\
\text { Toyota i-swing, Toyota I- } \\
\text { Unit, HAL Cyberdyne }\end{array}$ \\
\hline & & $\begin{array}{l}\text { ICT Enabled } \\
\text { Applications }\end{array}$ & $\begin{array}{l}\text { IT platforms, monitoring/ } \\
\text { tracking systems, Ambient } \\
\text { Intelligence, Proactivity }\end{array}$ \\
\hline & & Physical \& & Care service, supply with \\
\hline \multicolumn{2}{|c|}{ Digital } & $\begin{array}{l}\text { (forced by } \\
\text { embedded MST } \\
\text { and ICT) }\end{array}$ & $\begin{array}{l}\text { information, emergency } \\
\text { alert/call etc. }\end{array}$ \\
\hline
\end{tabular}

Figure 3. Implementation of of a new architectonic language for defining complex pervasive health environments
NASA also considers space vehicles and space stations equipped with various networked subsystems and intuitive and multimodal control interfaces as "ImmoBots" [18]. A common characteristic of "ImmoBots" is that they are able to control their internal subsystems autonomously for achieving certain goals, thus reducing mental stress of human beings interacting with them. The concept of the robotic service core is taking up this approach of autonomous and mental load reducing selfcontrol of complex networks of subsystems. Our goal is to support elderly people with case specific sets of subsystems of pervasive technologies in their home. Through the approach of distributing and integrating robotic systems into environments, the goal of the robotic service core is both outperforming the conventional smart home approach requiring still multiple control efforts and obtaining a higher acceptance of robotics as we have discussed (2.F. Cultural Challenges).

\section{Development Process}

For the creation of a value system that brings pervasive health and service technologies into the environment, and thus into the direct operating range of care receivers, their relatives and care givers, a multidisciplinary team for research and development is required. Various issues being relevant for the creation of a value system have been discussed (2. Challenges for Pervasive Healthcare). During the development of the service core concept, a main issue has been the continuous balancing of the requirements of the participating research fields derived from those challenges. The housing industry and related business models serving them have been placed in the center of the value system network. In a second phase, design rules for modules and technology sets of the service core component system have been formulated to simplify the implementation of knowledge of the participating research fields in the future. Moreover, the robotic service core states a first step to a cross faculty initiative for the creation of modular, scalable and ambient integrated pervasive health environments.

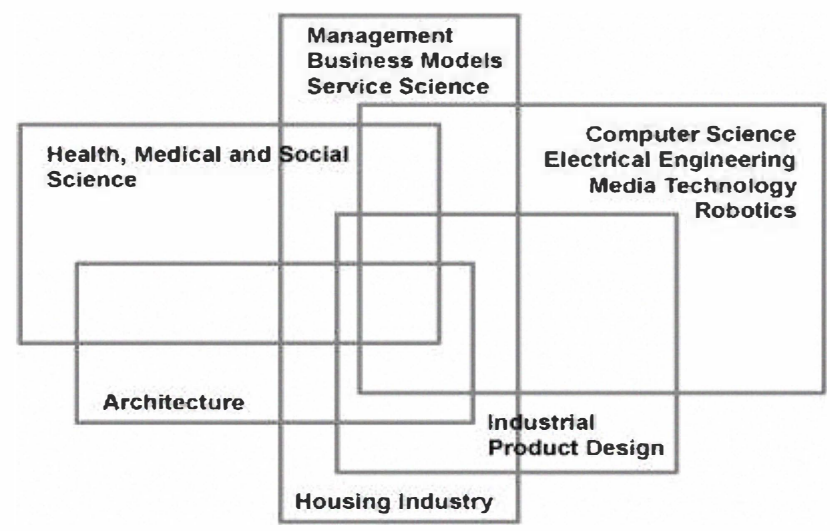

Figure 4. Participating fields of multidisciplinary Research and development to create a pervasive health value system

\section{System Integration}

As we have argued, middleware, while an important topic, in short has not finally been addressed in pervasive computing (2.D. Pervasive Computing Challenges). As we did not want to propose a novel system, we approached differently: by 
treating service cores composed of distributed sensing and actuation components as ImmoBots [18] we took the view of a robotic systems developer on distributed, heterogeneous, and communicating pervasive computing systems. Following the interdisciplinary approach that we argued for, we investigated the current state of the art of distributed sensing and actuation. The successful transfer and application of a robotics middleware Player [16] in the domain of pervasive computing has been already been shown [19]. Player has been the defacto standard and has been jointly developed for more than 10 years by the robotics community and reached a maturity far beyond other systems in pervasive computing. The successor middleware, ROS (Robot Operating System) [20], is downward compatible w.r.t. existing drivers and includes many modern concepts of distributed architectures. This includes decentralized peer-to-peer network concepts, publishsubscribe information distribution or bi-directional services between components. The middleware not only allows for inclusion of an immense variety of sensing and actuation systems, but also to visualize and simulate, both the information flow but also the physical space using e.g. OGRE (OpenSource 3D Graphics Engine) and ODE (Open Dynamics Engine) open source engines. This allows designing 3D objects in a CAD style manner, such as the different service cores, investigating their interaction and sending the very same information as the deployed sensor-actuator system would do - well before any physical prototyping is done. This reduces the time needed for iterative development and refinement and also costs. Also, the physical paths a human would have to take in such an environment can also be predicted, calculated and optimized already during the development phase in the middleware. This also has an impact on the prediction of the interaction times with the different digital systesm. The inclusion for real world simulation capabilities originates from e.g. robotic SLAM where algorithms have to be re-tested often but a real experiment is quite expensive and thus cannot be re-conducted for each run of a test.

The presented service cores have first been designed by an architect, together with an domain expert from assistive living, as CAD models. They would then be developed as virtual model using the proposed middleware and then be implemented as a physical scale model to allow and foster discussion with care receivers as end users. The proposed approach supports thereby also the development of "product configurator"-like tool to individualized components and functionality, prior to any deployment - similar to available online tools of a Swedish furniture manufacturers, while at the same time supports later mass production.

While in the simulation phase, the sensors have are modeled virtually, the real sensors send, by respectively their drivers, the same events and data as the simulation does. This enables us to develop assistive software on top of the respective cores and re-use it without any code modifications after the implementation of the scale model and finally in the 1:1 physical service core.

\section{E. System Overview}

We present the current state of the implementation and development process towards modular service cores for independent living. We show the integration into a demonstrator in Sec. IV. After the development of CAD models and pure simulation, we built a physical scale model allowing us to verify the utility and usability with care receivers, using it as technology probe [21]. The evaluation and discussion with focus groups are part of future work.

We introduce the functionality of the service core at the example of the scale model. While the sensors for a future service core unit will be different given the requirements on certification for health care technology, their data and generated events will be, by the employment of the discussed middleware, the same and finally, so will be the applications that can remain untouched. This is important to reduce the necessary efforts needed for the transfer of knowledge from model to the real world and it allows for parallelization of the development. Assistive applications can be developed while designers, architects and care givers discuss amount, size and human-exposed functionality. It is again important to state that the middleware allows substitution of sensors (from scale model to real world unit) without touching the application as long as the interface exposed by the sensor drivers is kept. For details on the concepts and implementation of ROS, the reader is referred to the documentation of the middleware [20].

The scale model (Figures 5 and 7) contains 12 motorized units for on demand available service functions, a touch sensor for each compartment, PIR sensors for general presence detection, and a ceiling mounted functional color camera for fall detection, several RFID readers and embedded tags in everyday objects, a wave sound output module for audio feedback, a scale model touch screen TV for convenient audio/visual communication with the care receiver, using multi-modal human-computer interaction accounting for potential deficiencies of the inhabitants. In the scale model various computer-human-interface devices have been experimentally implemented to test their applicability and to define solution spaces for fundamental communication challenges discussed (2.E. Social Challenges).

The middleware contains, besides drivers and abstraction layers from the specific instances of sensors and actuators, the control logic. Illegal states and actions are prohibited: the cooking plate cannot be hidden in the wall if there are pots present. Similar logic prevents systems to open if the care receiver is in too close proximity, or the bed hidden in the back wall (Figure 5, where the right hand is currently updating the system) cannot be extended if the sink on the left wall is used. This basic safety assessment is calculated based upon rules, such as the extent of a component into the room and an additional safety distance. In case of erroneous user requests, the system will either automatically retreat or close conflicting modules if the sensory systems detect no usage, or auditative or audio-visually warn the user and give supportive output using either the wave sound generator or the TV screen. Besides automatic rule checking, manual rules can be added by the designers, architects or system developers. 
The rules always will focus on maximum safety, as the target user group might not in all cases be aware on the consequences of their actions.

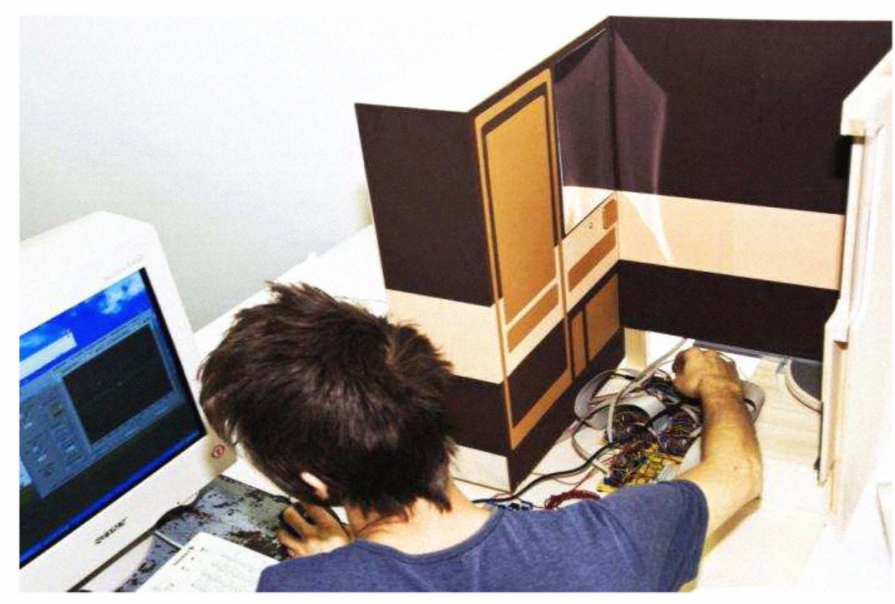

Figure 5. Fully functional experimental scale model and demonstrator, Scale 4:1. A room-in room type service core is shown. This example service core is an implementation of the functionality shown in Fig.2: bathroom, kitchen and sleeping room. The bed is hidden in the back wall, the bath room functionality is on the left wall behind and below the mirror, and the cooking functionality is in the left compartment of the left wall.

\section{THE DEMONSTRATOR}

We will now transfer the concept of robotic service cores into practice and introduce the physical scale model of an example service core. The employed pervasive computing middleware allows us to transfer the obtained results and insights and assistive pervasive health application software without modifications on the application side to a later 1:1 model.

The hardware platform used is the Arduino platform [22], a recent prototyping platform and toolkit, conceptually similar, e.g. with the notion of stackable add-on boards (so-called shields) to the well known Smart-Its platform that has been widely used in pervasive computing [23].

The complete sensing and control (see Figure 6) is realized via analog and digital sensors, such as the QProx QT110 touch sensors, Analog Devices ADXL345 accelerometers, Sharp's GP2Dx IR proximity sensors, Honeywell HMC6352 compass modules, RFID tagged devices (e.g. everyday objects used in the health assistance process) or a SEN-09334 JPEG camera with microcontroller compatible TTL level serial control. The selection of sensors reflects the required capabilities of the service modules to sense user presence, component state and orientation, and other physical properties of the environment and the user. Everyday objects (non-scale) use the Arduino Duemilanove base module. Sensor data is, if needed, wirelessly communicated via the Zigbee shield or alternatively via the Xport Ethernet shield. The overlay for the envisioned television overlay is generated by the TellyMate Video Output shield. In the scale model, a TouchShieldSlide, an 320x240 OLED resistive touch screen is used to simulate a scale model television device with $8 \mathrm{fps}$, but real video data.

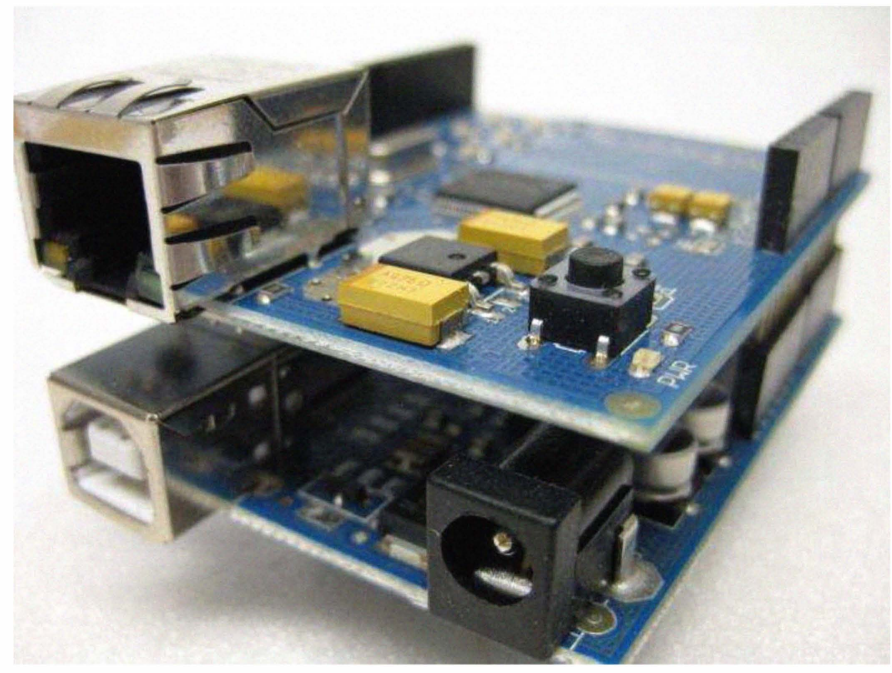

Figure 6. Close up of the Arduino Duemilanove with Ethernet Shield add-on, constituting the web server interface to the sensor and actor systems. The Arduino platform has been employed as it is a community-supported, open hardware and open software prototyping platform, allowing for customization and extension, supporting activity recognition and sensing in the service core.

\section{CONCLUSIONS, OUTLOOK AND FUTURE WORK}

We have discussed the multi-dimensional interdisciplinary challenges involved in the development and design process of pervasive healthcare technology.
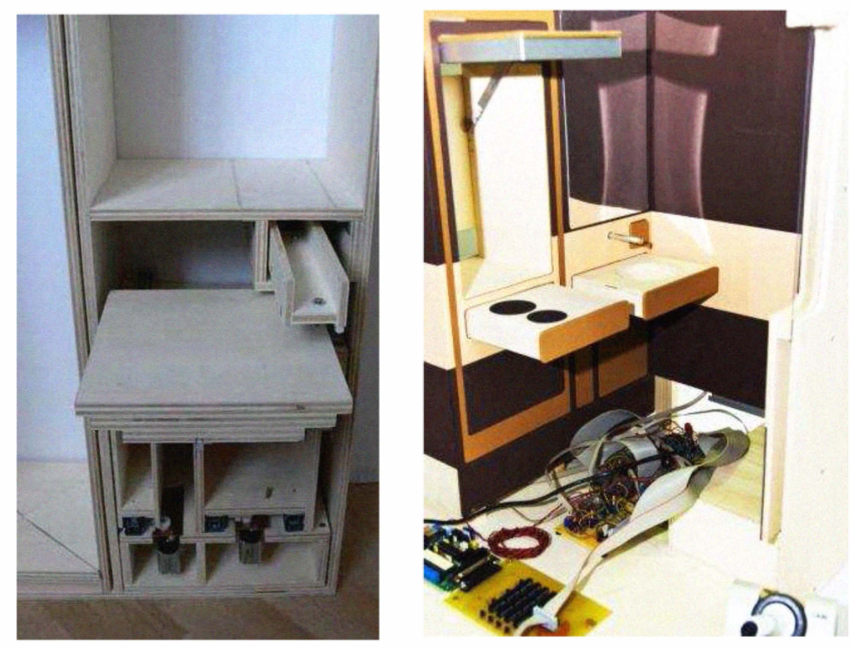

Figure 7. Left: 1:1 model of a Service Core "chassis", customizable through modular and compatible sets of functionalities, smart appliances, sensors, actors, design fit outs. All functions can be automatically retracted into the core unit. Right: Sensor and actuator-equipped scale model of a service core with a model kitchen compartment (left) and hygiene area with functional water supply and sink (right).

We have introduced the concept of modular service components as alternative approach to fully networked homes and houses and discussed the advantages of the proposed approach. The design process pursued has been reported on, and the relevant issues and challenges were discussed and situated in the body of related work. By the discussion of the employed middleware, facilitating simulation of both physical and digital operational sequences and workflows, and allowing for the separation and parallelism of sensing and actuation development, application development and deployment, we 
think that we have introduced ROS as successor of Player to the pervasive computing community by the presentation of a complete and technologically verified system.

Future work includes the modeling of human as "robots" in the middleware system, including the human motor apparatus. This will allow us to estimate interaction and movement times more precisely (time to walk from A to B, to grasp a physical object and perform pick and place tasks) and include this information in the development process to optimize computerhuman interaction. This will include the modeling of reduced sensitivity and mobility models of elderly people.

To verify the functionality and architecture, we intend to conduct user studies with care givers and receivers to optimize the selection of components for the individual service modules using the fully working scale model as technology probes.

As part of our future work, we will investigate how much and which information can be embedded into physical artifacts to support "embedded cognition", allowing in-place, in-situ task and health support for care receivers.

\section{ACKNOWLEDGEMENTS}

This work has been funded in parts by the German DFG funded Cluster of Excellence "CoTeSys - Cognition for Technical Systems."

\section{REFERENCES}

[1] Van den Acker, M., Buntinx, F., Metsemakers, J.F., Roos, S., Knottnerus, J.A.: Multimorbidity in general practice: prevalence, incidence, nd determin nts of co-occurring chronic and recurrent diseases, Pub Med J. Clin Epidemiol, vol 51(5), pp 367-375 May 1998

[2] VanderHart, G.P.: The Housing Decisions of Older Households: A Dynamic Analysis. Journal of Housing Economics, vol. 7, Issue 1, pp. 21-48, March 1998

[3] Linner, T., Bock, T.: Continuouss Customization in Architecture: towards customizable intelligent buildings. In Proceedings of MCPC2009 Conference on Mass Customization, Personalization nd Co-creation, Helsinki, Oktober 2009

[4] Bock, T., Linner, T.: Service Oriented Design. In proceedings of 2nd German Ambient Assisted Living Congress, Berlin, January 2009

[5] Kasanoff, B.: The Personal Economy. Keynote, MCPC2009 Conference on Mass Customization, Personalization nd Co-creation, Helsinki, Oktober 2009 - www.nowpossible.com: How to create markets for one person

[6] Habraken, N.J.: The Structure of the Ordinary - Form and Control in the Built Environment. The MIT Press, 2000. ISBN 0-262-08260-8.

[7] Initlle, S., Larson, K., Tapia, E.M., Beaudin, J., Kaushik, P., Nawyn, J., Rockinson, R.: Using a Live-in Laboratory for Ubiquitous Computing Research. Intl. Conf. on Pervasive Computing, pp. 349-365. 2006.

[8] Kidd, C.D., Orr, R., Abowd, G.D., Atkeson, C.G., Essa, I.A., MacIntyre, B., Mynatt, E.D., Starner, T., Newstetter, W.: The Aware Home: A Living Laboratoryy for Ubiquitous Computing Research. Cooperative Buildings. Integrating Information, Organziations nd Architecture. pp. 191-198. 1999. ISBN 978-3-540-66596-0
[9] Larson, K., Intille, S., McLeish, T.J., Beaudin, J., Williams, R.E.: Open Source Building - Reinventing Places of Living. BT Technology Journal, vol. 22(4), pp. 187-200. 2004.

[10] Torbensen, R.: OHAS: Open home automation system. IEEE Intl. Symposium on Consumer Electronics (ISCE), pp 1-4. 2008

[11] OpenRemote. Website, last visited 08.12.2009. http:/openremote.org/display/HOME/OpenRemote

[12] Holleis, P.: Integrating Usability Models into Pervasive Application Development. Ph.D. Thesis. Faculty of Mathematics, Computer Science and Statistics. LMU Munich. 2009.

[13] Consolvo, S., Roessler, P., Shelton, B.E., LaMarca, A., Schilit, B., Bly, S.: Technology for Care Networks of Elders. IEEE Pervasive Computing, vol 3(2), pp. 22-29. ISSN 1536-1268. 2004.

[14] Kranz, M., Schmidt, A., Maldonado, A., Rusu, B.R., Beetz, M., Hörnler, B., Rigoll, G.: Context-Aware Kitchen Utilities. Intl. Conference on Tangible and Embedded Interaction (TEI), pp. 213-214. 2007.

[15] Aitenbichler, E., Kangasharju, J., Mühlhäuser, M.: MundoCore: A lightweight infrastructure for Pervasive Computing. IEEE Pervasive and Mobile Computing, vol. 3 (4). pp. 332-361. ISSN 1574-1192. 2007

[16] Collet, T., MacDonald, B., Gerkey, B.: Player 2.0: Toward a Practical Robot Programming Framework. Proc. of the Australasi n Conf. on Robotics and Automation (ACRA). 2005.

[17] Román, M., Hess, C., Cerqueira R., Rang nath n, A., C mpbell, R.H., Nahrstedt, K.: Gaia: A Middleware Platform for Active Spaces. ACM SIGMOBILE Mobile Computing nd Communciations, vol 6 (4). pp 6567. ISSN 1559-1662. 2002

[18] Williams, B.C., Nayak, P.P.: Immobile Robots: AI in the New Millenium. AI Magazine, vol 17(3), pp. 16-35. 1996

[19] Kr nz, M., Schmidt, A., Rusu, R.B., Maldonado, A., Beetz, M., Hörnler, B., Rigoll, G.: Sensing Technologies and the Player-Middleware for Context-Awareness in Kitchen Environments. Intl. Conf. on Networked Sensing Systems. pp. 179-186. 2007.

[20] Quigley, M., Gerkey, B., Conley, K., Faust, J., Foote, T., Leibs, J., Berger, E., Wheeler, R., Ng, A.: ROS: an open-source Robot Operating System. Intl. Conference on Robotics and Automation. 2009.

[21] Hutchinson, H., Mackay, W., Westerlund, B., Bederson, B.B., Druin, A., Plais nt, C., Beaudoin-Lafon, M., Conversy, S., Evans, H., H nsen, H., Roussel, N., Eiderbäck, B.: Technology Probes: Inspiring design for and with families. Intl. Conf. on Hum n Factors in Computing Systems, pp. 17-24. ISBN 1-58113-630-7. 2003.

[22] Arduino Open Source Electronics Prototyping Plaform. Website, last visited 08.12.2009. http://www. arduino.cc

[23] Gellersen, H.W., Schmidt, A., Beigl, M.: Multi-Sensor ContextAwareness in Mobile Devices and Smart Artifacts. Mobile Networks and Applications, vol. 7(5), pp. 341-351. ISSN 1383-469X, 2002.

[24] Haus der Gegenwart. Munich, Germ ny. Partners: Microsoft, BMW, Munich City. Website, last visited 09.12.2009. http://www.haus-dergegenwart.de/partner/

[25] Intelligent Networking: T-Com House. Siemens, Telecom Laboratories. Berlin, 2005-2006

[26] Larson, K., Stephen, I. MIT Open Source Building Alliance - A house $n$ initiative. Position Paper, MIT House $n$, http://web media.mit.edu/ k11/AA OSBA\%20.pdf. 2003\#

[27] Shimizu, N.: A House of Sustainability: PAPI - Intelligent House in the Age of Ubiquitous Computing, in Architecture and Urbanism (AU), Special Issue Dec. 2005

[28] Roush, W.: Immobots take control. Technology Review, vol. J n 2003, pp. 36-41, 2003

[29] McC ndless, J.W., McC nn, R.S., Marshi, I. Kaiser, M.K., Andre,A.D. Human Factors Technologies for Space Exploration. In proceedings of Space 2006. San Jose, 2006 